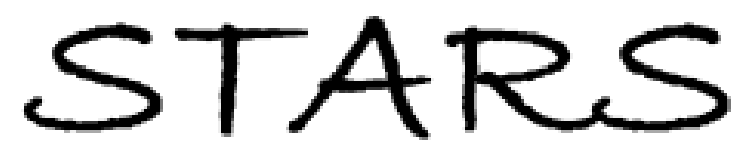

University of Central Florida

STARS

$1-1-2009$

\title{
Albedos of Main-Belt Comets 133p/Elst-Pizarro And 176p/Linear
}

Henry H. Hsieh

David Jewitt

Yanga R. Fernández

University of Central Florida

Find similar works at: https://stars.library.ucf.edu/facultybib2000

University of Central Florida Libraries http://library.ucf.edu

This Article is brought to you for free and open access by the Faculty Bibliography at STARS. It has been accepted for inclusion in Faculty Bibliography 2000s by an authorized administrator of STARS. For more information, please contactSTARS@ucf.edu.

\section{Recommended Citation}

Hsieh, Henry H.; Jewitt, David; and Fernández, Yanga R., "Albedos of Main-Belt Comets 133p/Elst-Pizarro And 176p/Linear" (2009). Faculty Bibliography 2000s. 1645.

https://stars.library.ucf.edu/facultybib2000/1645

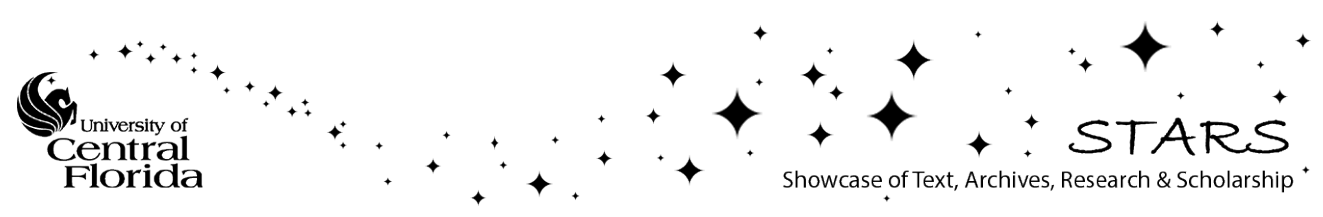




\title{
ALBEDOS OF MAIN-BELT COMETS 133P/ELST-PIZARRO AND 176P/LINEAR*
}

\author{
Henry H. Hsieh ${ }^{1}$, David JewitT ${ }^{2}$, and Yanga R. Fernández ${ }^{3}$ \\ ${ }^{1}$ Astrophysics Research Centre, Queen's University, Belfast, BT7 1NN, UK; h.hsieh@qub.ac.uk \\ ${ }^{2}$ Institute for Astronomy, University of Hawaii, 2680 Woodlawn Drive, Honolulu, HI 96822, USA; jewitt@ $@$ ifa.hawaii.edu \\ ${ }^{3}$ University of Central Florida, M.A.P. Building, 4000 Central Florida Blvd., Orlando, FL 32816, USA; yfernandez@physics.ucf.edu \\ Received 2008 November 20; accepted 2009 February 20; published 2009 March 10
}

\begin{abstract}
We present the determination of the geometric $R$-band albedos of two main-belt comet (MBC) nuclei based on data from the Spitzer Space Telescope and a number of ground-based optical facilities. For 133P/Elst-Pizarro, we find an albedo of $p_{R}=0.05 \pm 0.02$ and an effective radius of $r_{e}=1.9 \pm 0.3 \mathrm{~km}$ (estimated semiaxes of $a \sim 2.3 \mathrm{~km}$ and $b \sim 1.6 \mathrm{~km}$ ). For 176P/LINEAR, we find an albedo of $p_{R}=0.06 \pm 0.02$ and an effective radius of $r_{e}=2.0 \pm 0.2 \mathrm{~km}$ (estimated semiaxes of $a \sim 2.6 \mathrm{~km}$ and $b \sim 1.5 \mathrm{~km}$ ). In terms of albedo, 133P and $176 \mathrm{P}$ are similar to each other and are typical of other Themis family asteroids, C-class asteroids, and other comet nuclei. We find no indication that 133P and 176P are compositionally unique among other dynamically similar (but inactive) members of the Themis family, in agreement with previous assertions that the two objects most likely formed in situ. We also note that low albedo $\left(p_{R}<0.075\right)$ remains a consistent feature of all cometary (i.e., icy) bodies, whether they originate in the inner solar system (the MBCs) or in the outer solar system (all other comets).
\end{abstract}

Key words: comets: general - minor planets, asteroids

\section{INTRODUCTION}

The main-belt comets (MBCs), of which 133P/Elst-Pizarro (hereafter, 133P) and 176P/LINEAR (hereafter, 176P) are examples, occupy stable orbits that are decoupled from Jupiter and which are indistinguishable from the orbits of other mainbelt asteroids (Hsieh \& Jewitt 2006b). Dynamical simulations show that MBCs are extremely unlikely to originate in the Kuiper Belt given the current configuration of the major planets (e.g., Fernández et al. 2002), indicating that they are instead likely to be native to the main asteroid belt. Recent work suggests that some icy Kuiper Belt objects might have been delivered to the asteroid belt during the Late Heavy Bombardment (Levison et al. 2008), but even those simulations fail to produce the lowinclination, low-eccentricity orbits of MBCs such as 133P and $176 \mathrm{P}$.

In this Letter, we use observations from the Spitzer Space Telescope (hereafter, Spitzer; Werner et al. 2004) to determine the geometric albedos of $133 \mathrm{P}$ and $176 \mathrm{P}$, and then discuss the implications of these measurements.

\section{OBSERVATIONS}

We obtained optical observations of $133 \mathrm{P}$ and $176 \mathrm{P}$ on multiple occasions from 2003 through 2008 using the $10 \mathrm{~m}$ Keck I and University of Hawaii (UH) $2.2 \mathrm{~m}$ telescopes on Mauna Kea, and the $3.58 \mathrm{~m}$ New Technology Telescope (NTT) at the European Southern Observatory (ESO) at La Silla. Observations with the UH 2.2 m telescope were made using either a Tektronix $2048 \times 2048$ pixel CCD or the Orthogonal Parallel Transfer Imaging Camera (OPTIC; Tonry et al. 2004), both behind standard Kron-Cousins BVRI broadband filters.

\footnotetext{
* This work makes use of observations made with the Spitzer Space Telescope (Programs 3119 and 30678), which is operated by the Jet Propulsion Laboratory, California Institute of Technology under a contract with the National Aeronautics and Space Administration (NASA). Additionally, some data presented herein were obtained at the W. M. Keck Observatory that is operated as a scientific partnership among the California Institute of Technology, the University of California, and NASA, and was made possible by the generous financial support of the W. M. Keck Foundation. Some data presented herein were also obtained at ESO facilities at La Silla under program ID 081.C-0822(A).
}

Observations with Keck were made using the Low-Resolution Imaging Spectrometer (LRIS; Oke et al. 1995) in imaging mode. LRIS employs a Tektronix $2048 \times 2048$ CCD with standard Kron-Cousins BVRI filters. Observations with the NTT were made using the ESO Faint Object Spectrograph and Camera (EFOSC2; Buzzoni et al. 1984), which employs a $2048 \times 2048$ pixel Loral/Lesser CCD behind Bessel BVR broadband filters.

Bias subtraction and flat-field reduction were performed for all optical data. Dithered images of the twilight sky were used to construct flat fields for UH $2.2 \mathrm{~m}$ data, while images of the illuminated interior of the telescope dome were used to construct flat fields for Keck and NTT data. Photometry of our target objects and Landolt (1992) standard stars was obtained by measuring net fluxes within circular apertures of varying radii depending on the nightly seeing, with background sampled from surrounding circular annuli.

Spitzer observations of 133P (three visits, $166 \mathrm{~s}$ of total exposure time per visit; Figure 1(a)), using the $24 \mu \mathrm{m}$ channel (effective wavelength of $23.68 \mu \mathrm{m}$ ) of the Multiband Imaging Photometer for Spitzer (MIPS; Rieke et al. 2004) and originally obtained on 2005 April 11 as part of the Cycle 1 program 3119 (Reach et al. 2007), were retrieved from the Spitzer archive. Observations of 176P (two visits, $48 \mathrm{~s}$ of total exposure time per visit; Figure 1(b)), also with the $24 \mu \mathrm{m}$ channel of MIPS, were obtained on 2007 January 1 as part of the Cycle 3 program 30678. Observational circumstances are shown in Table 1. Photometry of our target objects from pipeline-processed Spitzer post-Basic Calibrated Data (PBCD) was obtained by measuring net fluxes within circular apertures with 6 pixel (14.'7) radii, and then applying appropriate aperture corrections (1.14 in the case of a 6 pixel aperture) and color corrections ( 0.96 for both targets).

\section{RESULTS}

We use our optical data to find best-fit IAU phase function parameters for $133 \mathrm{P}$ of $H_{R}=15.49 \pm 0.05 \mathrm{mag}$ and $G=0.04 \pm 0.05$, and best-fit linear phase function parameters (omitting data obtained at solar phase angles at which opposition surge effects are expected) of $m_{R}(1,1,0)=15.69 \pm 0.05 \mathrm{mag}$ 
Table 1

Spitzer Observations

\begin{tabular}{lcccccc}
\hline \hline Object & Date & $\mathrm{UT}$ & $R^{\mathrm{a}}$ & $\Delta_{\mathrm{Sp}}{ }^{\mathrm{b}}$ & $\alpha_{\mathrm{Sp}}{ }^{\mathrm{c}}$ & $m_{R}{ }^{\mathrm{d}}$ \\
\hline 133P/Elst-Pizarro & 2005 Apr 11 & $08: 01: 11$ & 3.596 & 3.046 & 14.6 & $21.56 \pm 0.15$ \\
& 2005 Apr 11 & $08: 04: 49$ & 3.596 & 3.046 & 14.6 & $21.63 \pm 0.15$ \\
& 2005 Apr 11 & $08: 08: 30$ & 3.596 & 3.046 & 14.6 & $21.70 \pm 0.15$ \\
176P/LINEAR & 2007 Jan 01 & $00: 49: 12$ & 3.162 & 2.541 & 16.2 & $20.58 \pm 0.11$ \\
& 2007 Jan 01 & $05: 22: 13$ & 3.163 & 2.539 & 16.1 & $20.21 \pm 0.11$
\end{tabular}

Notes.

${ }^{\text {a }}$ Heliocentric distance in AU.

${ }^{\mathrm{b}}$ Distance from Spitzer in AU.

c Solar phase angle (Sun-object-Spitzer) in degrees.

d Expected $R$-band magnitude as calculated from rotational-phase information inferred from the infrared data and observationally determined $H, G$ phase functions (Figure 2). Listed errors account for uncertainties in both rotational phase and phase function solutions.

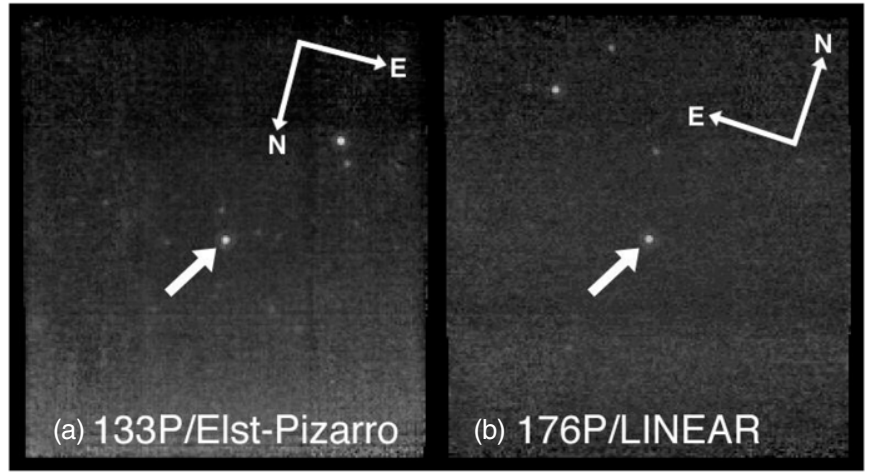

Figure 1. Composite (PBCD) $24 \mu \mathrm{m}$ images of (a) 133P/Elst-Pizarro (166 s total exposure time) and (b) 176P/LINEAR (48 s total exposure time), indicated by arrows, obtained using MIPS on Spitzer. Both objects are point sources with no indication of cometary activity. Each panel is $\sim 7^{\prime} .5$ by $8^{\prime} .2$ in size.

and $\beta=0.049 \pm 0.004 \mathrm{mag} \mathrm{deg}^{-1}$. These parameters are calculated using photometry obtained while 133P was observed to be inactive, and as such, are a refinement of parameters previously derived by Hsieh et al. (2004) from photometry obtained while $133 \mathrm{P}$ was visibly active. For $176 \mathrm{P}$, we find corresponding parameters of $H_{R}=15.10 \pm 0.05 \mathrm{mag}$, $G=0.26 \pm 0.05, m_{R}(1,1,0)=15.27 \pm 0.05 \mathrm{mag}$, and $\beta=0.034 \pm 0.005 \mathrm{mag} \mathrm{deg}^{-1}$. These parameters were likewise calculated only using photometry obtained while the comet was observed to be inactive. Plots of phase function solutions for both objects are shown in Figure 2. From their phase functions, we estimate our targets' expected mean optical magnitudes as viewed from Spitzer at the time of their observations to be $m_{R}=21.63 \mathrm{mag}$ for $133 \mathrm{P}$ and $m_{R}=20.39 \mathrm{mag}$ for $176 \mathrm{P}$.

Both objects exhibit significant rotational brightness variations, however, which represent significant sources of uncertainty in the interpretation of our infrared data. A rotation period of $P_{\text {rot }}=3.471 \mathrm{hr}$ and a lightcurve range of $\Delta m=0.4 \mathrm{mag}$ have been previously found for 133P (Hsieh et al. 2004). The rotational properties of $176 \mathrm{P}$ are currently poorly constrained. On 2007 March 21, however, we observed a photometric range for the object of $\Delta m \approx 0.6$ mag over $\sim 4.5 \mathrm{hr}$, suggesting a rotation period of $P_{\text {rot }} \geqslant 18 \mathrm{hr}$ (assuming a double-peaked lightcurve). This is consistent with Licandro et al. (2007b) who found $P_{\text {rot }}>22 \mathrm{hr}$.

Fortunately, constraints on the rotational phase of each object can be derived from the infrared data. Our second flux density measurement for $176 \mathrm{P}$ was 1.4 times larger than the first,

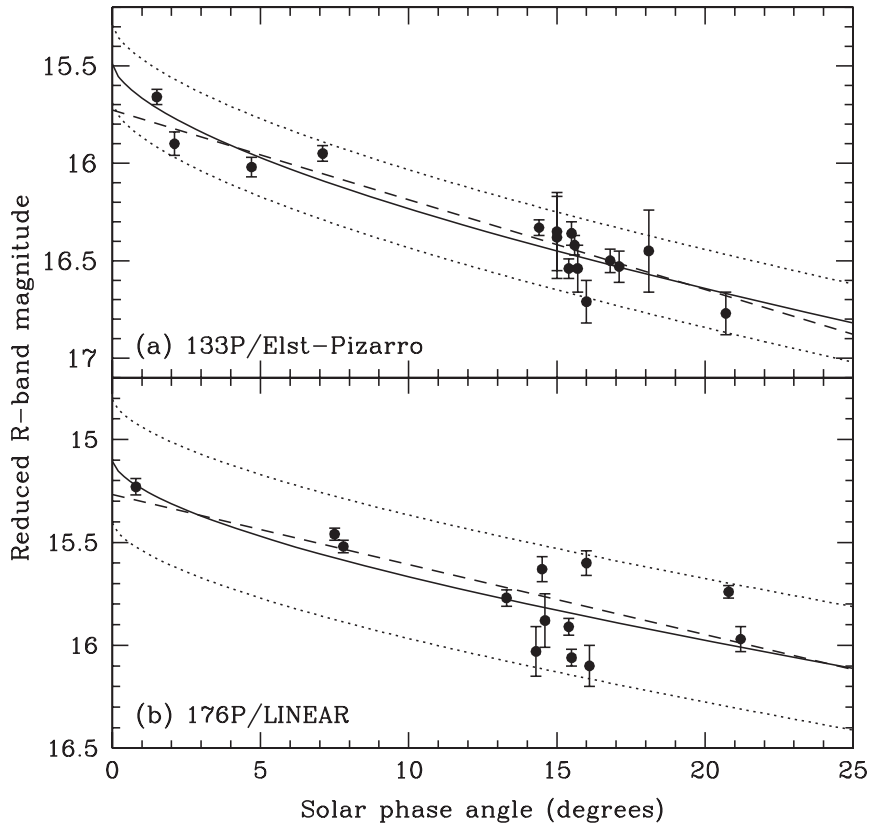

Figure 2. Phase function solutions for (a) 133P/Elst-Pizarro and (b) $176 \mathrm{P} /$ LINEAR, with best-fit IAU phase laws plotted as solid lines and best-fit linear phase functions plotted as dashed lines. Dotted lines indicate the expected range of brightness deviations from the IAU phase law due to rotation of the body. Observed reduced $R$-band magnitudes are plotted as solid circles.

implying an equivalent increase in visible cross-sectional area, corresponding to a change in visual magnitude of $\Delta m=$ $-0.37 \mathrm{mag}$, a significant fraction of the object's inferred optical photometric range. Such a large magnitude change indicates that the object was necessarily first observed near the minimum and then near the maximum of its lightcurve (consistent with the $4.55 \mathrm{hr}$ interval between the two observations). Using these constraints, we are able to adjust our optical brightness estimates (Table 1) accordingly, thereby reducing the effects of rotational phase uncertainty. The three Spitzer observations of 133P span only 7 minutes meaning that the rotational phase is less tightly constrained. Given 133P's short rotation period, however, a small decrease in the scattering cross section is still detectable between the first and last Spitzer observation, corresponding to a change in visual magnitude of $\Delta m=0.14$ mag and leading to the revised optical brightness estimates for 133P in Table 1.

We use the Harris (1998) Near-Earth Asteroid Thermal Model (NEATM) to iteratively solve for the effective radius, $r_{e}$, and geometric $R$-band albedo, $p_{R}$, of each object. As with any model, NEATM requires a number of assumptions, which, in turn, introduce uncertainties. One such source of uncertainty is the phase effect for thermal emission. NEATM treats the effect geometrically, calculating it based on the fraction of the Earth-facing hemisphere that is illuminated by the Sun at the time of observation. While this effect has been poorly measured and thus poorly constrained for large phase angles, the infrared phase coefficient of $0.01 \mathrm{mag} \mathrm{deg}^{-1}$ that we use here is generally considered to be appropriate for phase angles $\alpha<30^{\circ}$ (see Morrison 1977; Harris 1998). Thus, given the small phase angles $\left(14^{\circ}<\alpha<17^{\circ}\right)$ at which the Spitzer observations were obtained, this effect should introduce minimal systematic uncertainty into our calculations.

A more significant issue is that of the beaming parameter, $\eta$. We lack the minimum number of data points needed to constrain $\eta$ for either $133 \mathrm{P}$ or $176 \mathrm{P}$, forcing us to assume its value. A 
Table 2

Albedos and Radii Computed from Optical and Infrared Observations

\begin{tabular}{|c|c|c|c|c|c|c|c|c|c|}
\hline \multirow[t]{2}{*}{ Object } & \multicolumn{3}{|c|}{$\eta=0.8^{\mathrm{a}}$} & \multicolumn{3}{|c|}{$\eta=1.0^{\mathrm{a}}$} & \multicolumn{3}{|c|}{$\eta=1.2^{\mathrm{a}}$} \\
\hline & $F_{24 \mu m}$ & $r_{e}$ & $p_{R}$ & $F_{24 \mu m}$ & $r_{e}$ & $p_{R}$ & $F_{24 \mu m}$ & $r_{e}$ & $p_{R}$ \\
\hline \multirow[t]{3}{*}{ 133P/Elst-Pizarro } & $6.4 \pm 0.1$ & 1.78 & 0.054 & $6.5 \pm 0.1$ & 1.94 & 0.045 & $6.5 \pm 0.1$ & 2.09 & 0.039 \\
\hline & $6.0 \pm 0.1$ & 1.72 & 0.054 & $6.0 \pm 0.1$ & 1.88 & 0.045 & $6.1 \pm 0.1$ & 2.03 & 0.039 \\
\hline & $5.7 \pm 0.1$ & 1.67 & 0.054 & $5.7 \pm 0.1$ & 1.83 & 0.045 & $5.7 \pm 0.1$ & 1.97 & 0.039 \\
\hline \multirow[t]{2}{*}{ 176P/LINEAR } & $10.4 \pm 0.2$ & 1.72 & 0.068 & $10.5 \pm 0.2$ & 1.87 & 0.058 & $10.5 \pm 0.2$ & 2.01 & 0.050 \\
\hline & $14.6 \pm 0.2$ & 2.04 & 0.068 & $14.7 \pm 0.2$ & 2.22 & 0.058 & $14.7 \pm 0.2$ & 2.38 & 0.050 \\
\hline
\end{tabular}

Notes.

a Assumed $\eta$ value used to compute aperture- and color-corrected $24 \mu \mathrm{m}$ flux $\left(F_{24} \mu \mathrm{m}\right)$ in mJy (where uncertainties are estimated from sky background statistics), effective radius $\left(r_{e}\right)$ in $\mathrm{km}$, and geometric $R$-band albedo $\left(p_{R}\right)$

Spitzer survey of $\sim 50$ Jupiter-family comet nuclei by Fernández et al. (2008), however, found values of $0.6<\eta<1.2$, and all were consistent with $\eta \approx 0.94 \pm 0.20$. Given the results of this survey and assuming that $133 \mathrm{P}$ and $176 \mathrm{P}$ have low thermal inertias (similar to other comet nuclei, e.g., 9P/Tempel 1, which has $I<50 \mathrm{~W} \mathrm{~K}^{-1} \mathrm{~m}^{-2} \mathrm{~s}^{1 / 2}$; Groussin et al. 2007), we adopt $\eta=1.0$ as a reasonable assumption for solving for $r_{e}$ and $p_{R}$. To account for uncertainties in $\eta$, we also perform parallel calculations for $\eta=0.8$ and $\eta=1.2$ (Table 2).

Thus, assuming an emissivity of $\varepsilon=0.9$, we find $r_{e}=$ $1.9 \pm 0.3 \mathrm{~km}$ and $p_{R}=0.05 \pm 0.02$ for $133 \mathrm{P}$, and $r_{e}=2.0 \pm 0.2 \mathrm{~km}$ and $p_{R}=0.06 \pm 0.02$ for 176P. Estimated errors for both objects are mainly due to uncertainties in both $\eta$ and rotational phase. Given the observed photometric ranges $\left(\Delta m_{133 \mathrm{P}} \approx 0.40 \mathrm{mag} ; \Delta m_{176 \mathrm{P}} \approx 0.60 \mathrm{mag}\right)$ and corresponding inferred minimum axis ratios $\left([a / b]_{133 \mathrm{P}} \approx 1.45 ;[a / b]_{176 \mathrm{P}} \approx\right.$ 1.74) for each object, we find $a \sim 2.3 \mathrm{~km}$ and $b \sim 1.6 \mathrm{~km}$ for $133 \mathrm{P}$, and $a \sim 2.6 \mathrm{~km}$ and $b \sim 1.5 \mathrm{~km}$ for $176 \mathrm{P}$ as our best estimates of the semiaxes of each object.

\section{DISCUSSION}

We plot histograms showing the albedo $\left(p_{V}\right)$ distributions of several solar system body populations of interest in Figure 3. Assuming that both 133P and 176P are approximately spectrally neutral (i.e., $p_{V} \approx p_{R}$ ), based on 133P's spectral classification as a $\mathrm{C}$ - or B-type asteroid and 176P's classification as a B-type asteroid (Licandro et al. 2007b), we find that their albedos are typical of C-class asteroids (Figure 3(a)) and are also well within the distribution of albedos measured for members of the Themis asteroid family (Figure 3(b)), with which 133P and 176P appear to be dynamically associated (Hsieh \& Jewitt 2006b).

The Themis family is dominated by C-class asteroids (cf. Florczak et al. 1999), of which a substantial fraction (17 of the 39 currently classified members of the family, or $\sim 44 \%$ ) belong to the subclass of B-type asteroids. For comparison, B-type asteroids comprise only $\sim 15 \%$ of the general C-class population and $\sim 5 \%$ of all currently classified asteroids (Tholen \& Barucci 1989; Lazzaro et al. 2004). In terms of albedo, we find 133P and $176 \mathrm{P}$ to be consistent with both C-type asteroids and B-type asteroids (see Figures 3(a) and (d)), in agreement with their spectral classifications by Licandro et al. (2007b). Thus, in terms of both albedo and spectral type, 133P and 176P appear to be typical Themis asteroids, supporting previous speculation that the family might be home to more MBCs (Hsieh \& Jewitt 2006a).

Other objects such as $133 \mathrm{P}$ and $176 \mathrm{P}$ that also have orbits considered to be dynamically asteroidal, yet have been associated with observed or inferred cometary activity, are also classified as C-class objects. One such object is the crosslisted comet-

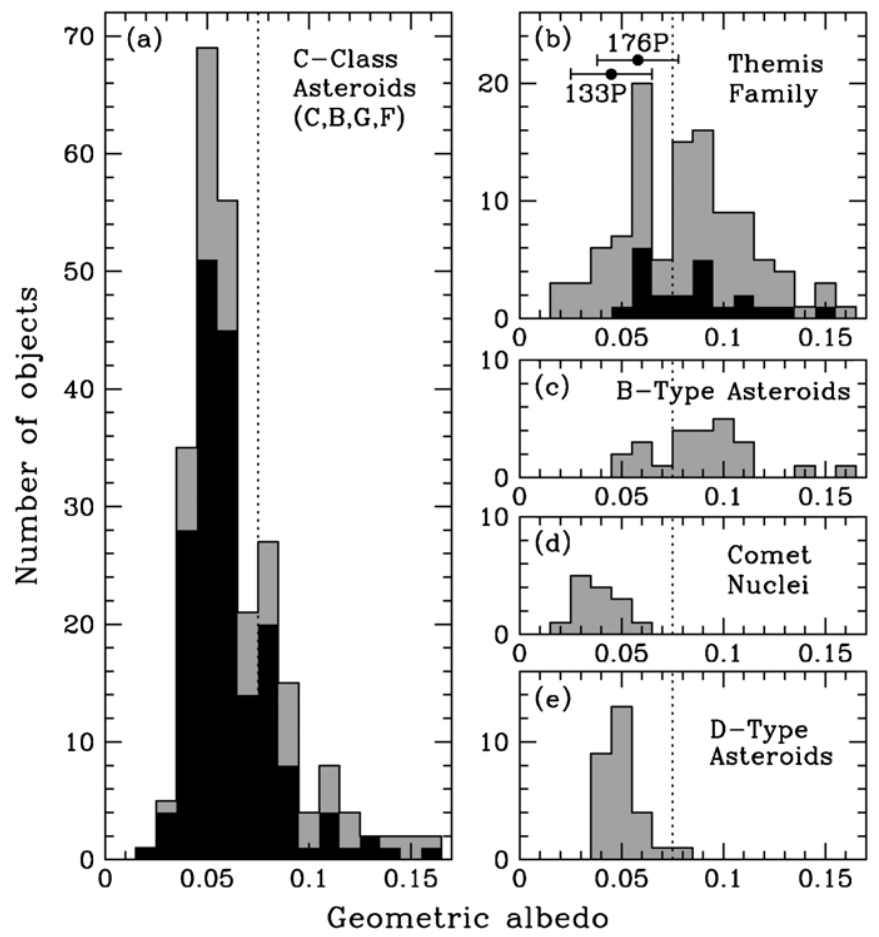

Figure 3. Histograms showing albedo distributions for (a) C-class (C-, B-, G-, or F-type) asteroids, where the superimposed black-shaded histogram only includes objects explicitly classified as C-type asteroids, (b) dynamical members of the Themis asteroid family, where the superimposed black-shaded histogram only includes those Themis members with measured albedos that have been classified as C-class asteroids (not all have been assigned taxonomic classes to date, however), (c) B-type asteroids, (d) active comet nuclei, and (e) D-type asteroids. All taxonomic classifications follow the Tholen system (Tholen \& Barucci 1989; Lazzaro et al. 2004). Comet nucleus albedos are from Lamy et al. (2004) and Brownlee et al. (2004), while all other albedo values are from the IRAS Minor Planet Survey (Tedesco et al. 2004). Objects with $p_{R}<0.075$, designated as "cometary" by Fernández et al. (2005), are to the left of the dotted lines.

asteroid 107P/(4015) Wilson-Harrington (C- or F-type; Tholen \& Barucci 1989). Other examples include the Geminid meteor stream parent 3200 Phaethon (B- or F-type; Tholen \& Barucci 1989; Licandro et al. 2007a) and its possible fragment, 155140 (2005 UD) (B- or F-type; Jewitt \& Hsieh 2006; Kinoshita et al. 2007). Another likely fragment of 3200 Phaethon, 1999 YC, appears spectrally neutral and is classified as a C-type object (Kasuga \& Jewitt 2008).

Being below the upper bound of "comet-like" albedos ( $p_{R}=$ 0.075) employed by Fernández et al. (2005), the albedos of $133 \mathrm{P}$ and $176 \mathrm{P}$ are also consistent with those of the nuclei of other active comets (Figure 3(d)). Spectroscopically, comet 
nuclei exhibit a broad range of colors, with both D-type-like and C-type-like spectral reflectivity gradients being found for various comets (see Fitzsimmons et al. 1994; Jewitt 2002), and in terms of albedos, the two MBC nuclei we consider here are consistent with both spectral types (Figures 3(a) and (e)). Thus, despite their strong dynamical association with mainbelt asteroids, we find that $133 \mathrm{P}$ and $176 \mathrm{P}$ have surfaces that may be compositionally comparable to other comets. This is consistent with Jewitt (2002) who suggested that the surface properties of short-period comet nuclei were likely largely due to sublimation-driven evolutionary effects and were not primordial in nature.

Studying the surface properties of the MBCs is vital for understanding their evolution and putting their volatile content into the proper context. In light of those goals, we find that, in terms of albedos, (1) $133 \mathrm{P}$ and $176 \mathrm{P}$ are similar to each other, (2) they are typical of other Themis asteroids and the C- and B-type asteroids that dominate the Themis family, and (3) their albedos are also consistent with albedos measured for other comet nuclei and D-type asteroids. Given these results, we find that low albedo continues to be a consistent feature of all cometary bodies, whether they originate in the outer or inner solar system. This finding necessarily also means, however, that albedo does not appear to be an effective diagnostic of the region from which a comet originates.

We acknowledge support of this work through STFC fellowship grant ST/F011016/1 to H.H.H., NASA Spitzer grant JPL1289078 and NASA Planetary Astronomy grant NNG05GF76G to D.J., and NASA grant JPL-1289123 to Y.R.F.. We also thank Bill Reach for valuable discussion and Alan Harris (DLR, Berlin) for pointing out an error in our initial albedo calculations.

\section{REFERENCES}

Brownlee, D. E., et al. 2004, Science, 304, 1764

Buzzoni, B., et al. 1984, The Messenger, 38, 9
Fernández, J. A., Gallardo, T., \& Brunini, A. 2002, Icarus, 159, 358

Fernández, Y. R., Jewitt, D. C., \& Sheppard, S. S. 2005, AJ, 130, 308

Fernández, Y. R., et al. 2008, LPI Contributions No. 1405, Paper ID. 8307

Fitzsimmons, A., Dahlgren, M., Lagerkvist, C. -I., Magnusson, P., \& Williams, I. P. 1994, A\&A, 282, 634

Florczak, M., Lazzaro, D., Mothé-Diniz, T., Angeli, C. A., \& Betzler, A. S. 1999, A\&AS, 134, 463

Groussin, O., et al. 2007, Icarus, 187, 16

Harris, A. W. 1998, Icarus, 131, 291

Hsieh, H. H., \& Jewitt, D. 2006, in IAU Symp. 229, Asteroids, Comets, Meteors, ed. D. Lazzaro, S. Ferraz-Mello, \& J. A. Fernández (Cambridge: Cambridge Univ. Press), 425

Hsieh, H. H., \& Jewitt, D. 2006, Science, 312, 561

Hsieh, H. H., Jewitt, D. C., \& Fernández, Y. R. 2004, AJ, 127, 2997

Jewitt, D. C. 2002, AJ, 123, 1039

Jewitt, D., \& Hsieh, H. 2006, AJ, 132, 1624

Kasuga, T., \& Jewitt, D. 2008, AJ, 136, 881

Kinoshita, D., et al. 2007, A\&A, 466, 1153

Lamy, P. L., Toth, I., Fernández, Y. R., \& Weaver, H. A. 2004, Comets II, ed. M. C. Festou, H. U. Keller, \& H. A. Weaver (Tucson, AZ: Univ. Arizona Press), 223

Landolt, A. U. 1992, AJ, 104, 340

Lazzaro, D., Angeli, C. A., Carvano, J. M., Mothé-Diniz, T., Duffard, R., \& Florczak, M. 2004, Icarus, 172, 179

Levison, H. F., Bottke, W. F., Nesvorný, D., Morbidelli, A., \& Gounelle, M. 2008, LPI Contributions No. 1405, Paper ID. 8156

Licandro, J., Campins, H., Mothé-Diniz, T., Pinilla Alonso, N., \& de León, J. 2007a, A\&A, 461, 751

Licandro, J., Pinilla Alonso, N., de León, J., Campins, H., Boehnhardt, H., Tozzi, G., Hainaut, O., \& Mothé-Diniz, T. 2007b, BAAS, 38, 470

Morrison, D. 1977, ApJ, 214, 667

Oke, J. B., et al. 1995, PASP, 107, 375

Reach, W. T., Kelley, M. S., \& Sykes, M. V. 2007, Icarus, 191, 298

Rieke, G. H., et al. 2004, ApJS, 154, 25

Tedesco, E. F., Noah, P. V., Noah, M., \& Price, S. D. 2004, IRAS Minor Planet Survey IRAS-A-FPA-3-RDR-IMPS-V6.0, NASA Planetary Data System, 12

Tholen, D. J., \& Barucci, M. A. 1989, Asteroids II, ed. R. P. Binzel, T. Gehrels, \& M. S. Matthews (Tucson, AZ: Univ. Arizona Press), 1139

Tonry, J. L., Burke, B. E., Luppino, G., \& Kaiser, N. 2004, Scientific Detectors for Astronomy, The Beginning of a New Era, ed. P. Amicor, J. W. Beletic, \& J. E. Beletic (Dordrecht: Kluwer), 300, 385

Werner, M. W., et al. 2004, ApJS, 154, 1 